\title{
EL PERFIL FORMATIVO Y PROFESIONAL DEL GRADUADO EN PEDAGOGÍA. PERSPECTIVA ACTUAL DE SU INSERCIÓN LABORAL.
}

\author{
Susana Tallón Rosales \\ Mirian Hervás Torres \\ M. Tamara Polo Sánchez \\ Carolina Fernández Jiménez \\ María Fernández Cabezas \\ mariafc@ugr.es
}

Fecha de Recepción: 9 Marzo 2018

Fecha de Admisión: 10 Abril 2018

\section{RESUMEN}

En el contexto actual y tras estos años de implantación del Plan Bolonia para la adecuación al Espacio Europeo de Educación Superior, es necesario revisar cómo ciertas disciplinas han afianzado su posición ante la sociedad. En este trabajo, nos vamos a centrar en la evolución del Grado de Pedagogía, así como del perfil profesional que desempeña, desde la perspectiva de los propios protagonistas, el alumnado, ya que serán en un futuro inmediato los profesionales que llevarán a cabo su labor en la sociedad, e implementarán aquellas competencias adquiridas, siendo los encargados de transmitir dicho legado. Por esta razón surge la necesidad de esta investigación, unido al hecho de que alrededor de la definición propia de la Pedagogía, existe ambigüedad y desconocimiento en torno a sus funciones y ámbitos de actuación profesional. Este estudio permitirá tener una visión contextualizada sobre los diferentes itinerarios laborales que esta profesión puede llevar a cabo, con la finalidad de extraer conclusiones sobre su perfil académico y por ende socio-laboral, favoreciendo una mejora de la imagen social del Pedagogo, la cual posibilite aminorar la incertidumbre existente respecto a este profesional. La metodología utilizada fue descriptiva y cuantitativa, con un diseño no experimental por método de encuesta. La muestra total 334 alumnos, pertenecientes al Grado de Pedagogía de la Universidad de Granada, matriculados en el curso académico 2016-2017. Los resultados obtenidos muestran que la figura del Pedagogo es un profesional multidisciplinar que puede abarcar diversos ámbitos de actuación y no sólo vinculado, al ámbito educativo, sino que se posibilita una apertura al contexto empresarial. Por ello, la formación (formal, no formal, y empresarial), es un pilar básico en nuestra sociedad. Ahí es, donde reside la importancia de la figura del Pedagogo, y cómo debe posicionarse como el experto en estas materias.

Palabras clave: pedagogía; educación superior; competencias; perfil profesional 


\section{EL PERFIL FORMATIVO Y PROFESIONAL dEL GRADUAdO EN PEDAGOGía. PERSPECTIVA ACTUAL DE SU INSERCIÓN LABORAL.}

\section{ABSTRACT \\ Training and professional profile of the graduate in pedagogy. Current perspective of their employment.}

After these years of implementation of the Bologna process for the adaptation to the European higher education area in the current context, it is necessary to review how certain disciplines have strengthened their position in society. In this paper, we will focus on the evolution of the degree of pedagogy, as well as professional profile which plays, from the perspective of the protagonists themselves, the students, since they will be professionals who carry out their work in the immediate future in the society, and will implement those acquired skills, being responsible for transmitting this legacy.

For this reason it is necessary of this research, coupled with the fact that around the definition of pedagogy, there is ambiguity and lack of knowledge regarding their functions and areas of professional activity. This study will allow having a vision contextualized on different working routes that this profession can carry out, in order to draw conclusions about its academic profile and therefore socio-labour, favoring an improvement of the image social of the pedagogue, which make it possible to reduce the uncertainty regarding this professional. The methodology used was descriptive and quantitative, with a non-experimental design by survey method. It shows total 334 students, belonging to the degree of pedagogy of the University of Granada, enrolled in the academic year 20162017. The results show that the figure of the pedagogue is a multidisciplinary professional who can cover different fields of action and not just linked to the field of education, but provides an opening to the business context. For this reason, the training (formal, non-formal, and business), is a basic pillar in our society. That is, where the importance of the figure of the pedagogue resides, and how it should position itself as the expert in these subjects.

Keywords: pedagogy; higher education; skills; professional profile

\section{INTRODUCCIÓN}

En el contexto actual sobre Educación Superior y los cambios de adaptación al europeo, se ha de destacar la singularidad de su recorrido histórico y su peso en la sociedad, de cada disciplina. En el caso de la Pedagogía, por ser una ciencia social de menor bagaje por su reciente implantación en comparación con otras, es necesario resaltar su particular situación. Como muchos autores indican, la Pedagogía, sus raíces continúan influenciando sobre su funcionalidad y desempeño laboral en nuestra sociedad. Tales argumentaciones giran en torno a su apego hacia la figura de cuidador, la escuela y su función docente, más que una ciencia que estudia todos los componentes del proceso de enseñanza-aprendizaje, atendiendo al contexto en el que dicho proceso se pueda dar, que no sólo ocurre en el ámbito educativo, sino también en contextos empresariales y no formales de la enseñanza.

En este contexto, urge la necesidad de consolidar el tránsito entre la Pedagogía clásica entendida como Paideia (ciencia del arte de enseñar, centrada en el acto docente de la infancia-juventud), hacia la Andragogía, (se enfatiza la concepción de que quien se educa es el hombre, y no sólo el niño). Este hecho, afecta a la imagen o definición en torno a esta profesión. El Grado de Pedagogía, está rodeado de cierta confusión 0 ambigüedad respecto a sus funciones, tanto que se confunde a menudo con la propia educación, la cual constituye en sí, un proceso, un hecho o una actividad concreta. En cambio, la Pedagogía ha de ser entendida como la disciplina, el estudio o el conjunto de normas, referidas a un hecho, a un proceso, 0 a una actividad de la educación. Por tanto, Pedagogía y Educación, no son sinónimos. La educación, es un hecho social, es tan antigua como el hombre, y la Pedagogía, es la ciencia de la educación, solo existe en la medida que alcanza su estatus cien- 
tífico. Por ello, la Pedagogía es un saber científico que teoriza, normativiza y legitima el hecho de la educación (García y López, 2014; Nassif, 1981; Riera y Civís, 2008).

El Pedagogo, está social y culturalmente vinculado a la función educativa. Hecho que perjudica su ampliación laboral hacia otros campos. Además, existe un potente intrusismo laboral, dado que otros perfiles no vinculados a la educación ejercen puestos de trabajo propios del profesional de la Pedagogía. Es por esta situación descrita, necesario clarificar el potencial y desarrollo laboral de la Pedagogía y del Pedagogo, y más aún por la importancia que tiene la educación en la sociedad por ser, el principal motor que promueve el cambio. Pero no sólo en el ámbito educativo, la Pedagogía se extiende hacia el mundo empresarial, por la importancia de la formación y la capacitación de los trabajadores. En esta línea, la Pedagogía Laboral, es la ciencia normativa que abarca el estudio de los problemas que el trabajo plantea a la educación. De ahí la necesidad, de incluir esta materia curricular dentro de los planes de estudio de Pedagogía, para atender los retos que demanda el mercado de trabajo respecto a la formación en organizaciones empresariales, y así, preparar al alumnado en la intervención en este ámbito profesional. Son varios los autores, que relacionan este ámbito empresarial con la Pedagogía, destacando la importancia de la presencia de contenidos de gestión de empresas y su relación con procesos educativos-formativos, que capaciten al Pedagogo a acceder a las empresas y ejercer su profesión. En dicho ámbito, se perfilan nuevas funciones del Pedagogo, destacadas a partir del Plan Bolonia, tales como, Asesor de docentes, Expertos de la educación y formación en espacios innovadores, Experto en orientación profesional, Asesor de empresa y Formador de formadores, entre otras (García y Aguilar, 2011; González, Martínez, y González, 2015; Pineda, 2002; Sánchez Morales, 2014; Viladot, 1992).

Atendiendo a la legislación, los Pedagogos actuarán en el ámbito del análisis, de la intervención y de la ejecución de sistemas y procesos educativos propios de la organización y administración del sistema educativo, en instituciones escolares y en agencias de educación no formal (Real Decreto 915/1992, regulador del título de Licenciado en Pedagogía). Tras la adaptación al Espacio Europeo de Educación Superior, tienen los estudiantes como finalidad la obtención de una formación general, orientada a la preparación para el ejercicio de actividades de carácter profesional. La flexibilidad y la diversidad son elementos sobre los que descansa la propuesta de ordenación de las enseñanzas oficiales (Real Decreto 1393/2007, de ordenación de enseñanzas oficiales universitarias).

En la actualidad, la evolución de estos estudios universitarios ha dado lugar a una apertura profesional respecto a sus campos de actuación. La propia Universidad de Granada ${ }^{1}$, expresa en su web corporativa que la titulación de Pedagogía capacita a los diferentes profesionales en sistemas, instituciones, contextos, recursos y procesos educativos y formativos, además de procesos de desarrollo personal, profesional, social y cultural., así llegará a ser especialistas en el diseño, gestión, desarrollo y evaluación de planes, proyectos, programas y acciones formativas y educativas adaptadas y contextualizadas, y en análisis, seguimiento y asesoramiento, realizando intervenciones educativas y formativas en contextos organizacionales y laborales.

La Universidad Autónoma de Barcelona, en su página oficial de Grado de Pedagogía ${ }^{2}$ (2018), indica que sus titulados están formados para trabajar en todos los contextos en los que haya personas en formación, ya sea por cuenta ajena o propia (Tabla 1). 


\section{EL PERFIL FORMATIVO Y PROFESIONAL dEL GRADUAdO EN PEDAGOGía. PERSPECTIVA ACTUAL DE SU INSERCIÓN LABORAL.}

Tabla 1.

Profesiones para las que capacita el Grado de Pedagogía, atendiendo a sus Ámbitos de Actuación Profesional.

\begin{tabular}{|c|l|}
\hline ÁMBITO & $\begin{array}{l}\text { Directores, técnicos y asesores pedagógicos de centros, asociaciones, } \\
\text { fundaciones... } \\
\text { Directores y gestores de proyectos, ocio, desarrollo humano sostenible } \\
\text { Mediadores socioeducativos y culturales } \\
\text { En áreas de educación en servicios sociales, personales, culturales, promoción } \\
\text { económica, justicia, políticas de educación, diversidad, equidad...tanto en } \\
\text { administraciones públicas como privadas }\end{array}$ \\
\hline ÁMBITO & $\begin{array}{l}\text { Coordinación de equipos, programas y servicios educativos } \\
\text { Diseño, desarrollo y evaluación de planes de formación } \\
\text { Impartición de formación y cursos } \\
\text { Orientar y asesorar a personas o grupos } \\
\text { Diseñar, producir materiales y recursos educativos } \\
\text { Dinamizar grupos y comunidades } \\
\text { Realizar estudios e investigaciones }\end{array}$ \\
\hline ÁMBITO & $\begin{array}{l}\text { Gestor, técnico y formador en departamentos de recursos humanos y } \\
\text { conocimiento } \\
\text { Orientadores profesionales y laborales } \\
\text { Productores y asesores pedagógicos en editoriales } \\
\text { Gestores de proyectos culturales y patrimoniales en centros cívicos, museos... } \\
\text { Diseñadores de programas educativos pedagógicos en medios de comunicación, } \\
\text { instituciones culturales y de investigación } \\
\text { Técnicos en procesos de innovación educativa y formativa }\end{array}$ \\
&
\end{tabular}

Fuente: Universidad Autónoma de Barcelona (2018).

Es en esta perspectiva de cambio y apertura a nuevas salidas profesionales e itinerarios laborales, donde el Pedagogo debe posicionarse, potenciar las posibilidades tan amplias de contextos y funciones que ofrece esta disciplina. En palabras de García Aguilera y Aguilar Cuenca (2011:15): "se trata de una titulación cada vez más transversal y aplicable a diferentes contextos de actuación, no exclusivos del ámbito puramente escolar". En ese sentido, la inserción laboral es el proceso que da paso de la inactividad a incorporarse al mercado de trabajo a través de la obtencion de un empleo. Es importante, que en dicho proceso de inserción laboral, los graduados aporten a los procesos de producción del mercado de trabajo, las competencias (conocimientos, destrezas y actitudes), adquiridas durante su periodo formativo (Aguilar Ramos, 2005; Vila, Dávila, y Mora, 2013).

El proceso de inserción laboral es cada vez más complejo, debido a la fuerte crisis económica que aún sigue afectando a nuestro país, frenando el progreso económico y social y aumentando las tasas de desempleo. En la actualidad, se alcanza la cifra de 3.470 .248 de desempleados. Respecto a los informes mensuales de mercado de trabajo de titulados universitarios, los datos recogidos para la titulación de Pedagogía, son 6.995 inscritos como demandantes de empleo, de los cuales 4.183 son parados, (hombres, 419 y mujeres, 3.764), atendiendo a los datos publicados por el Servicio Público de Empleo Estatal [SEPE] (2018) relativo al mes de Febrero de este año.

En relación a la información descrita en el Libro Blanco del Título de Pedagogía y Educación Social (2005:89):

Son contratados los recién titulados de Pedagogía, en su mayoría, en la empresa privada, concretamente un $60 \%$, del total de insertados laboralmente. Siendo las empresas públicas las siguientes llegando a un $28 \%$, y el resto, organizaciones o entidades sin ánimo de lucro. El promedio de recién titulados que encuentran trabajo relacionado con Pedagogía, lo consideran bajo. 
Es en el ámbito empresarial, donde el término competencia adquiere relevancia, puesto que responde a la capacidad de respuesta de los trabajadores ante nuevas situaciones complejas y cambiantes que debe superar en el día a día, para poner en práctica sus capacidades y conocimientos. En el ámbito académico-educativo, el término de competencia aparece con el Proceso Bolonia, a través de la especificación de las características de las titulaciones universitarias (Aznar, Cáceres, y Hinojo, 2011; Gimeno Sacristán, 2008). Entre los autores que destacan el campo empresarial, como uno más de sus ámbitos de actuación profesional, los autores Bordas, Cabrera, Fortuny, y Rodriguez-Lajo (1995), lo priorizan en su clasificación, donde el Pedagogo, se desarrolla en las siguientes siete categorías: (1) el mundo de la empresa, (2) la producción de materiales didácticos, (3) la enseñanza reglada, (4) la educación especial, (5) el tiempo libre y la educación sociocultural, (6) formación de formadores, y (7) la Pedagogía de ayer y de hoy. Es relevante que dicha clasificación está vigente desde hace más de veinte años. En dicho contexto de tiempo, otro autor resaltaba en su tesis doctoral, y respecto al papel del Pedagogo en la empresa, y la necesidad de mejorar sus competencias en dicho ámbito, la creación de una nueva especialidad "Pedagogía Empresarial", dentro del conjunto de las Ciencias de la Educación (Guillem Viladot, 1992).

En definitiva, para los profesionales de Pedagogía, es imprescindible especializarse en una rama, en palabras de Romero y Castellón (2016:30), con el objetivo de destacar en ese ámbito y aumentar las probabilidades de emplearse en puestos de trabajo acordes a su formación requerida y a la obtenida a través de su especialización. Tras estas conclusiones, respecto al mundo empresarial, y por ende a la Pedagogía Empresarial, por su compleja variedad de entidades e instituciones especializadas en ofrecer servicios formativos, es potencialmente, un ámbito ideal para el desarrollo profesional del Pedagogo y ampliar sus posibilidades de inserción laboral. Sin menospreciar el contexto educativo formal y no formal, hasta el momento, el ámbito más reconocido socialmente para estos profesionales.

\section{MÉTODO}

El objeto de esta investigación, consiste en dar a conocer los ámbitos profesionales donde pueden desempeñar sus funciones el Pedagogo, tanto ámbito no formal como empresarial, desde la perspectiva de los propios alumnos de esta titulación. Esta investigación atiende a una metodología descriptiva y cuantitativa, con un diseño no experimental por método de encuesta, al emplear como instrumento clave para la recogida de información, el cuestionario.

\section{PARTICIPANTES}

La muestra total fueron 334 alumnos, pertenecientes al Grado de Pedagogía de la Universidad de Granada, matriculados en el curso académico 2016-2017, pertenecientes a los cuatro cursos de la Facultad de Ciencias de la Educación. Se trata de un muestreo finito por conglomerados/estratos artificiales ya preelaborados. Es una muestra compensada en número de alumnos respecto a cada curso y respecto a la muestra mínima requerida por curso, superando los cuestionarios obtenidos en total. Si consideramos la variable género, obtenemos como resultado que la muestra no está compensada entre ambos sexos, ya que participaron 287 mujeres y 47 hombres.

\section{INSTRUMENTOS}

Se elaboró un Cuestionario ad hoc, estructurado en 3 bloques, (Datos Personales, Perfil del Pedagogo y Salidas Profesionales del Pedagogo) y 58 preguntas, de tipo cerradas, con cuatro alternativas de respuesta, desde totalmente de acuerdo a totalmente en desacuerdo, con una escala de Likert de graduación de respuesta de 1 a 4 . El tercer bloque a su vez, estructurado en 3 dimensiones, (Inserción Laboral, Ámbitos Profesionales de Actuación y Recursos Humanos). Para este tra- 


\section{El PERFIL FORMATIVO Y PROFESIONAL del GRADUAdo EN PEDAgogía. PERSPECTIVA ACTUAL DE SU INSERCIÓN LABORAL.}

bajo se ha empleado la dimensión "Ámbitos Profesionales de Actuación”, con 12 ítems. El Cuestionario, permite alcanzar una muestra de sujetos representativos de un colectivo amplio, y obtener mediciones cuantitativas (Buendía, Colás, y Hernández Pina, 1998; García, Alvira, Alonso, y Escobar, 2015).

\section{PROCEDIMIENTO}

Es una investigación que pretende abarcar los conocimientos y percepciones, que versan sobre la Pedagogía y el profesional que la desempeña, a través de la perspectiva del alumnado, por ello, durante el curso académico 20016-17, se llevó a cabo la pasación del cuestionario, mediante citas previamente concertadas con los docentes de los diversos grupos. Se procedía a entrar en el aula, en el tiempo asignado y distribuir los cuestionarios a todos los integrantes. Esta fase se llevó a cabo durante dos meses. A continuación, los análisis de las respuestas se fueron realizando mediante el paquete estadístico Statistical Package for the Social Sciences 22.0.

\section{RESULTADOS}

De los 12 ámbitos profesionales de actuación propuestos, que abarcan los tres campos de actuación profesional de los graduados en Pedagogía, todos han sido valorados en las opciones de respuesta positivas (totalmente de acuerdo y de acuerdo). Es decir, ningún ámbito es descartado 0 mal valorado. El alumnado asume y expresa su convencimiento de ello, a través de las puntuaciones. Por tanto, todos estos ámbitos forman parte de sus salidas profesionales, una vez graduados. El estudio de estadísticos descriptivos revela los tantos por cientos asignados por los alumnos en cada ámbito, siendo de los doce, los tres con mayor puntuación, ser orientador de un centro educativo, gestionar programas educativos para profesorado, alumnos y padres, y dedicarse a la inspección educativa (Tabla 2).

Tabla 2.

Resultados de los estadísticos descriptivos.

\begin{tabular}{|c|c|c|c|}
\hline Código & ITEMS & $\%$ & $\begin{array}{l}\text { Opción de } \\
\text { respuesta }\end{array}$ \\
\hline PGP & $\begin{array}{c}\text { Gestionar Programas Educativos en los Centros, } \\
\text { asesorando a Padres, Profesores y Alumnos }\end{array}$ & $66.80 \%$ & $\begin{array}{l}\text { Totalmente de } \\
\text { acuerdo }\end{array}$ \\
\hline POC & Ser el Orientador de un Centro Educativo & $71.90 \%$ & $\begin{array}{l}\text { Totalmente de } \\
\text { acuerdo }\end{array}$ \\
\hline PID & Impartir Docencia en Educación Secundaria & $34.40 \%$ & De acuerdo \\
\hline PFB & $\begin{array}{l}\text { Impartir Docencia en Formación Profesional y } \\
\text { Bachillerato }\end{array}$ & $37.40 \%$ & De acuerdo \\
\hline PDU & Impartir Docencia en la Universidad & $46.40 \%$ & $\begin{array}{l}\text { Totalmente de } \\
\text { acuerdo }\end{array}$ \\
\hline PRH & $\begin{array}{c}\text { Ejercer de Técnico de Recursos Humanos en Empresas y/o } \\
\text { entidades Públicas o Privadas }\end{array}$ & $40.40 \%$ & $\begin{array}{l}\text { Totalmente de } \\
\text { acuerdo }\end{array}$ \\
\hline PIE & Dedicarse a la Inspección Educativa & $49.10 \%$ & $\begin{array}{l}\text { Totalmente de } \\
\text { acuerdo }\end{array}$ \\
\hline PTH & Desempeñar su profesión en Hospitales & $44.30 \%$ & $\begin{array}{l}\text { Totalmente de } \\
\text { acuerdo }\end{array}$ \\
\hline PTE & $\begin{array}{c}\text { Realizar alguna labor en Empresas pertenecientes a } \\
\text { cualquier sector económico }\end{array}$ & $42.20 \%$ & De acuerdo \\
\hline PAF & $\begin{array}{c}\text { Ser básica su labor en Áreas de Formación y Educación en } \\
\text { entidades Públicas (Ayuntamiento, museos...) }\end{array}$ & $42.20 \%$ & De acuerdo \\
\hline PCP & $\begin{array}{l}\text { Trabajar en Centros Privados de Formación como } \\
\text { Formador }\end{array}$ & $57.60 \%$ & $\begin{array}{l}\text { Totalmente de } \\
\text { acuerdo }\end{array}$ \\
\hline PTP & Tener cabida en el ámbito de la Política & $32.60 \%$ & De acuerdo \\
\hline
\end{tabular}

Fuente: Elaboración Propia. 
Respecto al estudio de contingencias, cruzando los datos de los ítems con la variable Curso, para obtener datos respecto a diferencias o semejanzas por estar matriculado en un curso u otro del grado, se obtiene de los doce ámbitos (Tabla 3), un total de seis altamente significativos.

Tabla 3.

Resultados estudio de contingencia con variable Curso.

\begin{tabular}{c|c|c|c|c}
\hline \multirow{2}{*}{ ÍTEMS } & \multicolumn{5}{c}{ CURSO (CUR) } \\
\cline { 2 - 5 } & \multirow{2}{*}{ G.L. } & $\chi^{2}$ & P (SIG.) & $\begin{array}{c}\text { CASOS } \\
\text { VÁLIDOS }\end{array}$ \\
\hline PGP & 9 & $22,944^{\mathrm{a}}$ & $\mathbf{0 , 0 0 6 ^ { * * }}$ & 333 \\
\hline POC & 9 & $19,003^{\mathrm{a}}$ & $\mathbf{0 , 0 2 5 ^ { * * }}$ & 333 \\
\hline PID & 13 & $13,807^{\mathrm{a}}$ & 0,313 & 333 \\
\hline PFB & 9 & $10,947^{\mathrm{a}}$ & 0,279 & 333 \\
\hline PDU & 9 & $15,343^{\mathrm{a}}$ & 0,082 & 333 \\
\hline PRH & 9 & $15,825^{\mathrm{a}}$ & 0,071 & 333 \\
\hline PIE & 9 & $25,836^{\mathrm{a}}$ & $\mathbf{0 , 0 0 2 ^ { * * }}$ & 333 \\
\hline PTH & 9 & $25,526^{\mathrm{a}}$ & $\mathbf{0 , 0 1 1 ^ { * * }}$ & 332 \\
\hline PTE & 9 & $11,271^{\mathrm{a}}$ & 0,258 & 333 \\
\hline PAF & 9 & $36,011^{\mathrm{a}}$ & $\mathbf{0 , 0 0 0} * *$ & 333 \\
\hline PCP & 9 & $23,537^{\mathrm{a}}$ & $\mathbf{0 , 0 0 5 * *}$ & 333 \\
\hline PTP & 9 & $12,087^{\mathrm{a}}$ & 0,208 & 333 \\
\hline
\end{tabular}

Fuente: Elaboración Propia.

El ámbito de la gestión de programas educativos en centros, la implementación, desarrollo, asesoría a la comunidad educativa, y su evaluación, es considerado muy acorde al Pedagogo, dentro de un departamento de orientación (Tabla 4).

Tabla 4.

Resultado estudio de contingencia Ítem Un Pedagogo, puede: Gestionar programas educativos en los centros, asesorando a padres, profesores y alumnos, con la variable Curso.

\begin{tabular}{|c|c|c|c|c|c|c|}
\hline & \multicolumn{4}{|c|}{$\begin{array}{c}\text { UN PEDAGOGO, PUEDE: GESTIONAR PROGRAMAS } \\
\text { EDUCATIVOS EN LOS CENTROS, ASESORANDO A PADRES, } \\
\text { PROFESORES Y ALUMNOS (PGP) }\end{array}$} & \multirow[b]{2}{*}{ Total } \\
\hline & & $\begin{array}{c}\text { TOTALMENTE } \\
\text { DE } \\
\text { ACUERDO/ } \\
\text { EXCELENTE }\end{array}$ & $\begin{array}{l}\text { DE } \\
\text { ACUERDO/ } \\
\text { BUENA }\end{array}$ & $\begin{array}{l}\text { EN } \\
\text { DESACUERD } \\
\text { O/REGULAR }\end{array}$ & $\begin{array}{l}\text { TOTALMENT } \\
\text { E EN } \\
\text { DESACUER } \\
\text { DO/ } \\
\text { DEFICIENTE }\end{array}$ & \\
\hline \multirow[t]{4}{*}{ CURSO } & $1^{\circ}$ PEDAGOGIA & $21,6 \%$ & $6,3 \%$ & $2,4 \%$ & $1,8 \%$ & $32,1 \%$ \\
\hline & $2^{\circ}$ PEDAGOGIA & $15,0 \%$ & $2,7 \%$ & ,6\% & & $18,3 \%$ \\
\hline & $3^{\circ}$ PEDAGOGIA & $17,7 \%$ & $4,5 \%$ & ,6\% & $2,7 \%$ & $25,5 \%$ \\
\hline & $4^{\circ}$ PEDAGOGIA & $12,6 \%$ & $5,1 \%$ & $2,7 \%$ & $3,6 \%$ & $24,0 \%$ \\
\hline Total & & $67,0 \%$ & $18,6 \%$ & $6,3 \%$ & $8,1 \%$ & $100,0 \%$ \\
\hline
\end{tabular}

Fuente: Elaboración Propia.

El ámbito de la orientación dentro del contexto educativo, puntuada con la opción más a favor y en primer lugar (Tabla 5). 


\section{EL PERFIL FORMATIVO Y PROFESIONAL dEL GRADUAdO EN PEDAGOGía. PERSPECTIVA ACTUAL DE SU INSERCIÓN LABORAL.}

Tabla 5.

Resultado estudio de contingencia Ítem Un Pedagogo puede: ser el orientador del centro educativo, con la variable Curso.

\begin{tabular}{|c|c|c|c|c|c|c|}
\hline & \multicolumn{4}{|c|}{$\begin{array}{c}\text { UN PEDAGOGO, PUEDE: SER EL ORIENTADOR DEL CENTRO } \\
\text { EDUCATIVO (POC) }\end{array}$} & \multirow[b]{2}{*}{ Total } \\
\hline & & $\begin{array}{c}\text { TOTALMENTE } \\
\text { DE } \\
\text { ACUERDO/ } \\
\text { EXCELENTE }\end{array}$ & $\begin{array}{c}\text { DE } \\
\text { ACUERDO/ } \\
\text { BUENA }\end{array}$ & $\begin{array}{c}\text { EN } \\
\text { DESACUERD } \\
\text { O/REGULAR }\end{array}$ & $\begin{array}{c}\text { TOTALMENT } \\
\text { E EN } \\
\text { DESACUER } \\
\text { DO/ } \\
\text { DEFICIENTE }\end{array}$ & \\
\hline \multirow[t]{4}{*}{ CURSO } & $1^{\circ}$ PEDAGOGIA & $23,4 \%$ & $3,9 \%$ & $2,7 \%$ & $2,1 \%$ & $32,1 \%$ \\
\hline & $2^{\circ}$ PEDAGOGIA & $15,9 \%$ & $1,8 \%$ &, $3 \%$ &, $3 \%$ & $18,3 \%$ \\
\hline & $3^{\circ}$ PEDAGOGIA & $18,6 \%$ & $3,3 \%$ & $1,2 \%$ & $2,4 \%$ & $25,5 \%$ \\
\hline & $4^{\circ}$ PEDAGOGIA & $14,1 \%$ & $3,3 \%$ & $3,0 \%$ & $3,6 \%$ & $24,0 \%$ \\
\hline Total & & $72,1 \%$ & $12,3 \%$ & $7,2 \%$ & $8,4 \%$ & $100,0 \%$ \\
\hline
\end{tabular}

Fuente: Elaboración Propia.

El ámbito de la inspección educativa cuyo acceso es mediante concurso-oposición y necesario unos años específicos con un cargo de funcionario previamente, obtiene por parte de los alumnos muy buena puntuación (Tabla 6).

Tabla 6.

Resultado estudio de contingencia Ítem Un Pedagogo puede: dedicarse a la Inspección Educativa, con la variable Curso.

\begin{tabular}{|c|c|c|c|c|c|c|}
\hline & \multicolumn{4}{|c|}{$\begin{array}{c}\text { UN PEDAGOGO, PUEDE: DEDICARSE A LA INSPECCIÓN } \\
\text { EDUCATIVA (PIE) }\end{array}$} & \multirow[b]{2}{*}{ Total } \\
\hline & & $\begin{array}{c}\text { TOTALMENTE } \\
\text { DE } \\
\text { ACUERDO/ } \\
\text { EXCELENTE }\end{array}$ & $\begin{array}{c}\text { DE } \\
\text { ACUERDO/ } \\
\text { BUENA }\end{array}$ & $\begin{array}{c}\text { EN } \\
\text { DESACUERD } \\
\text { O/REGULAR }\end{array}$ & \begin{tabular}{|c} 
TOTALMENT \\
E EN \\
DESACUER \\
DO/ \\
DEFICIENTE \\
\end{tabular} & \\
\hline \multirow[t]{4}{*}{ CURSO } & $1^{\circ} \mathrm{PEDAGOGIA}$ & $17,4 \%$ & $10,2 \%$ & $3,9 \%$ &, $6 \%$ & $32,1 \%$ \\
\hline & $2^{\circ}$ PEDAGOGIA & $9,3 \%$ & $7,8 \%$ & $1,2 \%$ & & $18,3 \%$ \\
\hline & $3^{\circ}$ PEDAGOGIA & $11,4 \%$ & $10,2 \%$ & $1,8 \%$ & $2,1 \%$ & $25,5 \%$ \\
\hline & $4^{\circ}$ PEDAGOGIA & $11,1 \%$ & $5,7 \%$ & $3,9 \%$ & $3,3 \%$ & $24,0 \%$ \\
\hline Total & & $49,2 \%$ & $33,9 \%$ & $10,8 \%$ & $6,0 \%$ & $100,0 \%$ \\
\hline
\end{tabular}

Fuente: Elaboración Propia.

El ámbito hospitalario con una puntación favorable alrededor del $76 \%$ de los alumnos, destaca la función de atención educativa y formativa a personas en edad de escolarización, que por motivos de enfermedad no pueden acudir a un centro escolar (Tabla 7). 
Tabla 7.

Resultado estudio de contingencia Ítem Un Pedagogo, puede: desempeñar su profesión en hospital, con la variable Curso.

\begin{tabular}{|c|c|c|c|c|c|c|}
\hline & \multicolumn{4}{|c|}{$\begin{array}{l}\text { UN PEDAGOGO, PUEDE: DESEMPEÑAR SU PROFESIÓN EN } \\
\text { HOSPITALES (PTH) }\end{array}$} & \multirow[b]{2}{*}{ Total } \\
\hline & & $\begin{array}{c}\text { TOTALMENTE } \\
\text { DE } \\
\text { ACUERDO/ } \\
\text { EXCELENTE }\end{array}$ & $\begin{array}{c}\text { DE } \\
\text { ACUERDO/ } \\
\text { BUENA }\end{array}$ & $\begin{array}{c}\text { EN } \\
\text { DESACUERD } \\
\text { O/REGULAR }\end{array}$ & \begin{tabular}{|c|} 
TOTALMENT \\
E EN \\
DESACUER \\
DO/ \\
DEFICIENTE
\end{tabular} & \\
\hline \multirow[t]{4}{*}{ CURSO } & $1^{\circ}$ PEDAGOGIA & $15,7 \%$ & $9,0 \%$ & $6,0 \%$ & $1,5 \%$ & $32,2 \%$ \\
\hline & $2^{\circ}$ PEDAGOGIA & $8,7 \%$ & $6,6 \%$ & $2,1 \%$ & ,6\% & $18,1 \%$ \\
\hline & $3^{\circ}$ PEDAGOGIA & $11,4 \%$ & $9,9 \%$ & $1,8 \%$ & $2,4 \%$ & $25,6 \%$ \\
\hline & $4^{\circ}$ PEDAGOGIA & $8,7 \%$ & $6,3 \%$ & $7,2 \%$ & $1,8 \%$ & $24,1 \%$ \\
\hline Total & & $44,6 \%$ & $31,9 \%$ & $17,2 \%$ & $6,3 \%$ & $100,0 \%$ \\
\hline
\end{tabular}

Fuente: Elaboración Propia.

En el mundo empresarial, las funciones de un Pedagogo quedan enmarcadas a los departamentos de formación y a los de Recursos Humanos, como más relevantes. Los alumnos encuestados consideran a este ámbito muy interesante para su desarrollo profesional y amplitud de posibilidades de inserción laboral (Tabla 8).

Tabla 8.

Resultado estudio de contingencia Ítem Un Pedagogo puede: ser básica su labor en áreas de formación y educación en entidades públicas, con la variable Curso.

\begin{tabular}{|c|c|c|c|c|c|c|}
\hline & \multicolumn{4}{|c|}{$\begin{array}{l}\text { NN PEDAGOGO, PUEDE: SER BÁSICA SU LABOR EN ÁREAS DE } \\
\text { FORMACIÓN Y EDUCACIÓN EN ENTIDADES PÚBLICAS (PAF) }\end{array}$} & \multirow[b]{2}{*}{ Total } \\
\hline & & $\begin{array}{c}\text { TOTALMENTE } \\
\text { DE } \\
\text { ACUERDO/ } \\
\text { EXCELENTE }\end{array}$ & $\begin{array}{c}\text { DE } \\
\text { ACUERDO/ } \\
\text { BUENA }\end{array}$ & $\begin{array}{c}\text { EN } \\
\text { DESACUERD } \\
\text { O/REGULAR }\end{array}$ & $\begin{array}{c}\text { TOTALMENT } \\
\text { E EN } \\
\text { DESACUER } \\
\text { DO/ } \\
\text { DEFICIENTE }\end{array}$ & \\
\hline \multirow[t]{4}{*}{ CURSO } & $1^{\circ}$ PEDAGOGIA & $11,7 \%$ & $13,8 \%$ & $6,0 \%$ &, $6 \%$ & $32,1 \%$ \\
\hline & $2^{\circ}$ PEDAGOGIA & $9,3 \%$ & $5,1 \%$ & $3,9 \%$ & & $18,3 \%$ \\
\hline & $3^{\circ}$ PEDAGOGIA & $13,2 \%$ & $7,8 \%$ & $2,1 \%$ & $2,4 \%$ & $25,5 \%$ \\
\hline & $4^{\circ}$ PEDAGOGIA & $9,0 \%$ & $7,2 \%$ & $3,3 \%$ & $4,5 \%$ & $24,0 \%$ \\
\hline Total & & $43,2 \%$ & $33,9 \%$ & $15,3 \%$ & $7,5 \%$ & $100,0 \%$ \\
\hline
\end{tabular}

Fuente: Elaboración Propia.

El ámbito privado, en concreto, centros de formación, de refuerzo académico, de preparación de oposiciones, y organismos relacionados con formación superior privada que ofrezca posgrados y másteres. La labor de formador, constituye una salida profesional asumida por mayoría por el $80 \%$ de los participantes (Tabla 9.) 


\section{EL PERFIL FORMATIVO Y PROFESIONAL dEL GRADUAdO EN PEDAGOGía. PERSPECTIVA ACTUAL DE SU INSERCIÓN LABORAL.}

Tabla 9.

Resultado estudio de contingencia Ítem Un Pedagogo puede: trabajar en centros privados de formación como formado, con la variable Curso.

\begin{tabular}{|c|c|c|c|c|c|c|}
\hline & \multicolumn{4}{|c|}{$\begin{array}{c}\text { UN PEDAGOGO, PUEDE: TRABAJAR EN CENTROS PRIVADOS } \\
\text { DE FORMACIÓN COMO FORMADOR (PCP) }\end{array}$} & \multirow[b]{2}{*}{ Total } \\
\hline & & $\begin{array}{c}\text { TOTALMENTE } \\
\text { DE } \\
\text { ACUERDO/ } \\
\text { EXCELENTE }\end{array}$ & $\begin{array}{c}\text { DE } \\
\text { ACUERDO/ } \\
\text { BUENA }\end{array}$ & $\begin{array}{c}\text { EN } \\
\text { DESACUERD } \\
\text { O/REGULAR }\end{array}$ & \begin{tabular}{|l} 
TOTALMENT \\
E EN \\
DESACUER \\
DO/ \\
DEFICIENTE \\
\end{tabular} & \\
\hline \multirow[t]{4}{*}{ CURSO } & $1^{\circ}$ PEDAGOGIA & $19,8 \%$ & $8,1 \%$ & $3,0 \%$ & $1,2 \%$ & $32,1 \%$ \\
\hline & $2^{\circ}$ PEDAGOGIA & $10,8 \%$ & $6,0 \%$ & $1,2 \%$ &, $3 \%$ & $18,3 \%$ \\
\hline & $3^{\circ}$ PEDAGOGIA & $16,2 \%$ & $5,7 \%$ & $1,2 \%$ & $2,4 \%$ & $25,5 \%$ \\
\hline & $4^{\circ}$ PEDAGOGIA & $10,5 \%$ & $7,5 \%$ & $1,5 \%$ & $4,5 \%$ & $24,0 \%$ \\
\hline Total & & $57,4 \%$ & $27,3 \%$ & $6,9 \%$ & $8,4 \%$ & $100,0 \%$ \\
\hline
\end{tabular}

Fuente: Elaboración Propia.

En definitiva, para concluir este apartado, destacar que son significativas las diferencias de opiniones entre los distintos grupos, con el hecho de ser primero de Pedagogía quien muestra mayoría de respuestas positivas en comparación con el resto de cursos. Respecto a los ámbitos profesionales de actuación, en relación a su perfil, inserción laboral y funciones, no muestran confusión, consideran a grandes rasgos los ámbitos laborales descritos como parte o podrían serlo, de su desarrollo profesional, teniendo la formación y recursos adecuados.

\section{DISCUSIÓN}

Tras los datos mostrados, se ratifica la existencia de lagunas en la formación del alumnado de Pedagogía, puesto que se demanda una formación más práctica y acorde a la realidad del mercado de trabajo. De los diversos ámbitos mostrados que se consideran propios para la Pedagogía y que el alumnado así lo cree, es necesario la introducción de materias curriculares o reforzamiento de las mismas, sobre todo del ámbito no Formal y Empresarial. Respecto a los Ámbitos de Actuación, manifiestan que el entorno educativo-formativo es el más acorde, 0 al menos donde han visto la labor de algún Pedagogo o Pedagoga, en entidades públicas de formación, en Universidades, y en centros específicos de educación. La impartición de la docencia, a medida que la etapa educativa es superior, más acorde se perciben, siendo ésta en la Universidad la más puntuada.

En el contexto formativo, atendiendo a entidades públicas, organismos e instituciones de carácter no formal, valoran positivo ejercer sus funciones en ámbitos como inspección educativa, formación en hospitales, y áreas de educación, y asesoría política. El Pedagogo es un profesional que trabaja continuamente con la legislación educativa y además debe mantenerse actualizado. Por tanto un Pedagogo, como especialista en educación, sistemas y procesos, hace que sea la persona adecuada para asesorar, dentro estructuras políticas. En el contexto empresarial, como sector emergente, consideran que ejercer su labor en empresas de cualquier sector, es parte de sus salidas profesionales, tales como, la formación impartida en centros privados, y la gestión recursos humanos.

En resumen, se puede concluir que el Pedagogo es un profesional que puede desarrollar su labor en diferentes contextos (ej. Escolar, social, cultural, empresarial). En el proceso de inserción laboral de los jóvenes titulados y concretamente de los graduados en Pedagogía, ante la singularidad por el tradicional enfoque de la intervención en el ámbito escolar, surgen otros nuevos que pretenden dar respuesta educativa-formativa a contextos no escolarizados. En este sentido, la empresa es un ámbito de actuación profesional más, para los Pedagogos. Con toda esta investigación, se 
puede conceptualizar el perfil del Profesional de la Pedagogía en la actualidad, y su acceso al mercado laboral. Destacar, que queda mucho por hacer e investigar sobre la Pedagogía y el Pedagogo, con la intención de contribuir a mejorar su posicionamiento en la sociedad.

\section{REFERENCIAS}

Agencia Nacional de Evaluación de la Calidad y Acreditación . (2005). Libro Blanco Título de Grado en Pedagogía y Educación Social. Madrid: Aneca.

Aguilar Ramos, M. (2005). La Inserción Laboral de los Jóvenes en España. Un enfoque microeconómico. Navarra: Editorial Aranzadi, S.A.

Aznar, I., Cáceres, M., y Hinojo, M. (2011). La adquisición de competencias específicas en la Educación Superior. Evaluando la formación del psicopedagogo en la Universidad de Granada. Ensayos, Revista de la Facultad de Educación de Albacete, 26, 71-93.

Bordas, I., Cabrera, F., Fortuny, M., y Rodriguez-Lajo, M. (1995). Les sortides professionals del Ilicenciat en Pedagogía. Barcelona: Universitat de Barcelona Publicacions.

Buendía, L., Colás, M., y Hernández Pina, F. (1998). Métodos de investigación en Psicopedagogía. Madrid: McGraw-Hill.

García Aguilera, F., y Aguilar Cuenca, D. (2011). Competencias Profesionales del Pedagogo. Ámbitos laborales y nuevos yacimientos de empleo. Málaga: Aljibe, S.L.

García, M., Alvira, F., Alonso, L., y Escobar, M. (2015). El análsiis de la Realidad Social. Métodos y técnicas de investigación. Madrid: Alianza Editorial.

Gimeno Sacristán, J. (2008). Educar por competencias, ¿qué hay de nuevo? Madrid: Ediciones Morata.

González Lorente, C., Martínez Clares, P., y González Morga, N. (2015). El perfil formativo del Graduado en Pedagogía: La visión del Alumnado. . Profesorado. Revista de Curriculum y formación del profesorado, 19(1), 394-412.

Ministerio de Educación y Ciencia. (1992). Real Decreto 915/1992 de 17 julio, por el que se establece el titulo universitario oficial de Licenciado de Pedagogía. Madrid: Boletin Oficial del Estado.

Ministerio de Educación y Ciencia. (2007). Real Decreto 1393/2007, de 29 de octubre, por el que se establece la ordenación de las enseñanzas universitarias oficiales. Madrid: Boletín Oficial del Estado.

Nassif, R. (1981). Pedagogía General. Madrid: Cincel.

Pineda, P. (2002). Pedagogía Laboral. Barcelona: Ariel Educación .

Riera i Romani, J., y Civis i Zaragoza, M. (2008). La Pedagogía Profesional del siglo XXI. Educación $X X I, 11,133-154$.

Romero Rodríguez, J., y Castelló Quintana, A. (2016). Redefiniendo los campos de inserción laboral del Pedagogo. Curriculum, 29, 21-34.

Sánchez Morales, P. (2014). El papel de la Pedagogía en la Formación Empresarial. Pedagogy role in Business Training. Cuestiones Pedagógicas, 23, 85-104.

SEPE. (2018). Servicio Público de Empleo Estatal. Recuperado de https://www.sepe.es/contenidos/que_es_el_sepe/estadisticas/datos_avance/pdf/empleo/evolparo.pdf

Servicio Público de Empleo Estatal. (2018). Informes y estudios sobre tendencias de mercado de trabajo de personas tituladas. Recuperado 1de https://www.sepe.es/contenidos/observatorio/titulaciones/2018_02/titulados/ciencias_sociales_y_juridicas/ESTUDIO_TITULADOS_FI_6.pdf

Universidad de Granada. (2018). Titulaciones. Grado en Pedagogía. Recuperado de http://grados.ugr.es/pedagogia 


\section{EL PERFIL FORMATIVO Y PROFESIONAL DEL GRADUAdO EN PEDAGOGíA. PERSPECTIVA ACTUAL DE SU INSERCIÓN LABORAL.}

Universitat Autónoma de Barcelona. (2018). Catálogo e información de las Titulaciones. Grado en Pedagogía. Recuperado de http://www.uab.cat/web/estudiar/listado-de-grados/informaciongeneral/pedagogia-1216708258897.html?param1=1228291018203

Vila, L., Dávila, C., y Mora, J. (2013). Cómo promover la adquisión de competencias para la innovación en la Universidad. La visión de los graduados de las universidades de Latinoamérica. En J. Mora, H. Schomburg y U. Teicher, Empleo y Trabajo de los graduados universitarios. (pp. 189208). Barcelona: Ediciones Octaedro, S.L.

Viladot, G. (1992). La proyección profesional del Pedagogo en la empresa. Apuntes para una Pedagogía Empresarial. Barcelona: Dpto. Pedagogía Aplicada, Bellaterra.

1 Consulta realizada en Internet en (. http://grados.ugr.es/pedagogia), Marzo 2018.

2 Consulta realizada en internet. Universidad Autónoma de Barcelona. Marzo 2018. http://www.uab.cat/web/estudiar/listado-de-grados/informacion-general/x1216708258897.html?param1=1228291018203 